\title{
Review
}

\section{Spinal cord injury and neurogenic lower urinary tract dysfunction: what do we know and where are we going?}

\author{
William Dodd ${ }^{1}$, Kartik Motwani ${ }^{1}$, Coulter Small ${ }^{1}$, Kevin Pierre ${ }^{1}$, Devan Patel $^{1}$, \\ Samuel Malnik ${ }^{1}$, Brandon Lucke-Wold ${ }^{1, *}$, Ken Porche $^{1}$ \\ ${ }^{1}$ Department of Neurosurgery, University of Florida, Gainesville, FL 32601, USA \\ *Correspondence: brandon.luckewold@gmail.com (Brandon Lucke-Wold) \\ Submitted: 17 September 2021 Accepted: 20 October 2021 Published: 19 January 2022
}

\begin{abstract}
One of the well reported but difficult to manage symptoms of spinal cord injury (SCI) is neurogenic lower urinary tract dysfunction (NLUTD). The type of NLUTD is variable based on location and extent of injury. SCI affects more males and NLUTD is especially debilitating for men with incomplete injury. This review summarizes the anatomical basis of NLUTD in SCI and discusses current diagnostic and management strategies that are being utilized clinically. The last two sections address new innovations and emerging discoveries with the goal of increasing scientific interest in improving treatment options for people with SCI. Areas warranting further investigation are pinpointed to address current gaps in knowledge and/or appropriate technology.
\end{abstract}

Keywords: Spinal cord injury; Neurogenic lower urinary tract dysfunction; Diagnostics; Neurostimulation; Emerging discoveries

\section{Background}

Spinal cord injury (SCI) can affect sensory, motor, and autonomic tracts leading to neurogenic lower urinary tract dysfunction (NLUTD) [1]. These people often are para and/or tetraplegics depending on injury location and men are more commonly affected. Elderly men are more likely to have incomplete SCI and suffer from increased urologic dysfunction due to comorbidities [2]. The associated NLUTD is often rated as most disruptive to overall quality of life. Men with SCI and NLUTD are at increased risk for infections, incontinence, vesicoureteral reflux, nephrolithiasis, and renal failure [3]. These patients require multidisciplinary efforts for effective diagnosis and treatment [4]. An important understanding of anatomical basis of injury progression is necessary. The extent of injury ranges from complete to incomplete with the American Spinal Injury Association (ASIA) scale most commonly employed [5]. The primary functions of the bladder are to store and empty urine. These functions require a complex interplay between the central and peripheral nervous systems. Consequently, the manifestations of NLUTD in patients with SCI depend on the location of the injury. Given that spinal control of micturition occurs at the S2-S4 elements of the sacral plexus, urinary manifestations of SCI can be broadly categorized as suprasacral, sacral, and infrasacral based on the level of the injury. Suprasacral lesions are upper motor neuron lesions that result in detrusor hyperactivity, resulting in detrusor overactivity [6]. Additionally, patients can develop neurogenic acontractile detrusor when pathology affects at or above this level. Lower urinary tract dysfunction is characterized by simultaneous hyperactivity of both the detrusor muscle and external sphinc- ter [7]. This results in the bladder contracting against a closed sphincter. The resulting obstruction can cause reflux of urine into the ureters and kidneys, ultimately placing patients at an increased risk for urinary tract infections, renal disease, and increased bladder pressure [7].

The spinal level of SCI causes distinct neuroanatomical disruption patterns resulting in different treatment strategies. Suprasacral lesions produce neurogenic detrusor overactivity (NDO) and a noncentralized sacral spinal reflex (detrusor-sphincter dyssynergia; DSD). Sacral lesions directly involve the spinal micturition center and cause mixed NLUTD symptoms and detrusor underactivity [8]. Pudendal nerve lesions result in small bladder volumes and urinary frequency [9]. Infrasacral spinal cord injuries result in lower motor neuron disease. Patients can develop neurogenic acontractile detrusor resulting in overflow incontinence due to lack of bladder contractions [8]. Conus medullaris lesions are more likely to cause urinary retention and/or incontinence due to detrusor areflexia and decreased external urethral sphincter tone. Even among male patients with similar pathogenic injuries there remain individual differences in symptomology, degree of SCI, and thus optimal treatment options. Understanding these differences is paramount to best practice as well as interpreting the efficacy of novel experimental treatments.

\section{Workup}

Prompt evaluation of lower urinary tract function is important considering its cause of increased morbidity and mortality and high prevalence in SCI (70\%-84\%) male victims. Clinical patterns can help determine the presence of NLUTD and help localize the lesion to guide con- 
servative, pharmacologic, and surgical therapies. Several guidelines exist for evaluating NLUTD including European Urological Association, Joint Societe Internationale D'Urologie-International Consultation on Urologic Diseases (SIU-ICUD), and Japanese clinical guidelines [1012]. Consensus for male patients is summarized below.

Workup begins with a thorough history and physical exam especially with focus on male urologic history regarding benign prostate hypertrophy, urinary infrequency, and any urologic malignancies. Review of the initial trauma and severity of the SCI with a validated system like the ASIA score is first performed. It is important to consider factors in a male's medical history, like severe hypertension or history of acute angle closure glaucoma, that may contraindicate the use of medications in the treatment in NLUTD [13]. A bladder diary may provide information on number of voids and/or straight catheterizations, voiding times, volume voided, amount of fluid ingested, feelings of urgency, and episodes of incontinence [14]. This is especially important for males with incomplete SCI. The use of a voiding diary in combination with questions of history, onset, associated symptoms, pain, chronicity, and presence of urgency, hesitancy, frequency, dysuria, sensation of bladder filling, hematuria, discharge, and pain, may help delineate the cause and serve as a baseline to determine treatment effect. Physical exam should also include assessment of the bulbo-cavernosus reflex, anal reflex, voluntary pelvic floor contractions, anal sphincter tone, and sensation of the sacral dermatomes. Evaluation of the prostate is also important in males. Diagnostic laboratory studies such as urinalysis, kidney function studies, blood glucose, and prostate specific antigen can be useful in rule out other conditions.

Bladder function can be objectively evaluated using urodynamic studies at least once within the months following injury and can measure bladder sensitivity. For incomplete SCI, this can help determine return of function. Video urodynamics serve as the gold standard for those with NLUTD. It is generally recommended to perform the first urodynamic study 3-6 month following injury. Electromyography may evaluate external urethral sphincter function. The resulting morphologies based on the information gained regarding intravesicular pressure, detrusor activity, bladder compliance, bladder sensitivity, and bladder storage capacity can help diagnose NLUTD, differentiate between the different subtypes, such as those that involve detrusor overactivity or DSD, localize the level of SCI, identify the extent of injury on ASIA scale, give prognosis, and monitor treatment effect.

Post-void residuals can be measured using bladder ultrasound or clean intermittent catheterization. Other nonneurogenic conditions can be ruled out using renal and pelvic ultrasonography. MRI or CT of the brain can rule out other neurogenic causes, particularly in the setting of polytrauma where the brain may be affected and cause disruption of the pontine micturition center. MRI of the spine can reveal spinal cord compromise that may be amenable to surgical intervention with possible subsequent symptom improvement [15]. This is especially important for older males where cervical fractures are common due to underlying diffuse idiopathic skeletal hyperostosis is common.

Dilation of the ureters, calyxes, and hydronephrosis should be considered in those with NLUTD. The upper urinary tract can be evaluated using ultrasound and magnetic resonance urography [16]. Several classification systems exist for urinary tract dysfunction for those with NLUTD as mentioned previously and key workup options were discussed above.

\section{Current treatments}

\subsection{Guiding principles}

SCI is unique from other NLUTD etiologies in that it is often accompanied by traumatic injuries and spinal shock syndrome. These factors create distinct phases of treatment for urologic issues affecting males: the acute recovery phase, post-acute rehabilitation phase, and long-term management phase [17]. Allowing time for resolution before establishing baseline urodynamics and other tests can prevent the need for repeat procedures and ensure optimal treatment strategy selection, especially in males with incomplete SCI. Self-reported measures (SRMs), a bladder diary (frequency-volume chart), urinary tract imaging, and validated surveys (e.g., NLUTD symptom score, Qualiveen) can assist in setting up the best long-term care for SCI patients.

\subsection{Conservative management strategies and pharmacological treatments}

SCI patients may require indwelling catheterization during the acute recovery phase until renal and cardiovascular function is optimized and urodynamic parameters improved [17]. Beyond the acute phase, clean intermittent catheterization (CIC) is the standard method for patients with insufficient bladder emptying [18]. Permanent transurethral or suprapubic catheters are less favorable due to increased risk of lower urinary tract infections [19]. External condom catheters can be used in male patients with incontinence or those that use reflex voiding, however the compressive force of the device on the penis can cause penile injury and ischemia, especially in patients with dementia or decreased extremity sensation [20]. This is an important issue to consider in older males with incomplete cervical SCI.

Pharmacological treatments of NLUTD generally target neuromuscular junctions in the smooth muscle of the detrusor or internal urethral sphincter. Antagonism of alpha-1 adrenergic receptors relaxes the internal sphincter and bladder neck to improve voiding. Terazosin and tamsulosin are two of the most well-studied $\alpha 1$ antagonists and both have been demonstrated to reduce autonomic dysreflexia and improve urodynamics, especially in males $[21,22]$. Antago- 
nism of muscarinic acetylcholine receptors in the detrusor decreases the frequency and urgency associated with neurogenic detrusor overactivity. A meta-analysis of $16 \mathrm{RCTs}$ totaling 960 patients showed anti-muscarinics improved bladder filling and patient-reported outcomes compared to placebo [23]. The same study found no significant differences between oxybutynin, which has the most supporting data, and other anticholinergic agents [20]. Mirabegron, a beta-3 adrenergic receptor agonist, also decreases detrusor activation and has shown promising improvements in urgency and frequency when used as a second-line treatment after antimuscarinic medications [24]. Mirabegron also has the advantage of a more favorable side effect profile, but is not likely to overtake antimuscarinics as the first-line treatment until more large RCTs are available [25].

Chemodenervation of the detrusor by botulinum neurotoxins (BoNT) has also emerged as a minimally invasive treatment for NLUTD in men with incomplete SCI. The most commonly used BoNT serotype is onabotulinumtoxin A (BoNT/A), commercially known as Botox (Allergan, Irvine, Ca.) [26]. Although the bulk of the literature is pertaining to nonneurogenic overactive bladder, there have been several high-quality placebo-controlled trials of BoNT/A in NLUTD patients. Regardless of etiology, intradetrusor injection of BoNT/A is a safe and effective method for reducing neurogenic detrusor overactivity symptoms. Targeted engineering of novel botulinum-like toxins could further improve this treatment modality [26].

\subsection{Neurostimulation and surgical interventions}

Neurostimulation via implantation of externallycontrolled electrodes at the sacral nerve roots may be considered in cases where conservative management fails to adequately control symptoms or produces unfavorable side effects. The Brindley Procedure, combined sacral anterior root stimulation (SARS) and rhizotomy of dorsal sacral roots S1-S5 can be considered in NLUTD patients where conservative management fails [27]. Deafferentiation of the dorsal roots decreases detrusor reflex contraction which facilitates proper bladder filling. Anterior root stimulation can then encourage timely micturition by stimulating detrusor contraction. The external urethral sphincter may also be stimulated by SARS; however, pulsatile stimuli permit micturition between electrical impulses because the striated sphincter relaxes more rapidly than the smooth muscle of the detrusor. Continence, bladder capacity, incidence of UTI, and male-reported quality of life are all improved after the Brindley procedure, though it is important to note many patients continue anticholinergic and CIC treatments after incomplete SCI [28-30]. The drawbacks of the Brindley procedure are infection of the implanted equipment, need for revision procedures, and the permanent nature of the dorsal rhizotomy. Sacral deafferentiation alone has also been explored as an alternative in patients who are not eligible candidates for SARS; however, the data do not currently support it as a standard of care $[31,32]$.

\section{Engineering innovations}

As outlined above, bladder storage and micturition are controlled by a complex interplay between the smooth muscle of the lower urinary tract, the striated muscle of the urethral sphincter, the cerebral cortex, pontine micturition center, sacral micturition center, and both the sympathetic and parasympathetic portions of the peripheral nervous system. In incomplete SCI, the range of damage is variable, and, therefore, may affect different elements of these cascades, which explains the different etiologies and presentations of NLUTD after SCI. Despite this complexity, a simplified view of NLUTD after SCI may be described as improper sensory awareness for the need to void or improper stimulus delivery to cause the bladder to void. Therefore, new treatment options have focused on the use of sensors to monitor bladder pressure parameters, neuromodulation to deliver precise stimulation to cause the micturition cascade, and combined closed-loop neuromodulation systems to both sense bladder fullness and void. Some of the key findings were highlighted above in current treatments section but the experimental approaches are addressed further below and may be especially valuable for males with SCI. Outline of promising approaches highlighted in Table 1 (Ref. [33-39]).

Urodynamic testing is a known study that examines the parasympathetic and sympathetic function of the urinary system, enabling examiners to evaluate bladder and urinary sphincter function and integrity [40]. Clinical utility is highlighted in the workup section. Urodynamic testing requires specialized equipment and trained operators, as such, it is usually performed in large hospitals and is relatively expensive, limiting its utility for patients with SCI [34]. New innovations focus on increasing the portability and enabling real-time data collection to assess the detrusor pressure. Increasing portability and reliability is necessary to increase the utility of testing across a broader clinical spectrum [33]. Real time data from implantable sensors would allow users to know when to void and function much like the afferent neurons that provide information about bladder fullness in those without SCI [34]. This should only be done for men with bladder anesthesia and incomplete SCI. Although some limitations exist with the complexity of implantation and device accuracy, one study found no significant difference in bladder pressure monitoring between a novel portable device and traditional urodynamic testing [41].

Many of the recent innovations have been as a result of improved targeting of electrical stimulation and noninvasive modalities [37]. Traditionally, neuromodulation was performed using sacral anterior root stimulation to stimulate voiding; however, accompanying rhizotomy was necessary to treat neurogenic detrusor overactivity and DSD [29]. The associated rhizotomy was eschewed by some pa- 
Table 1. New innovations for managing urinary dysfunction following SCI.

\begin{tabular}{ll}
\hline Goal & Strategy \\
\hline \multirow{3}{*}{ Enhanced diagnosis and monitoring } & - Utilize long term ambulatory devices to track urodynamic measurements \\
& over time [33] \\
& - Develop portable, wireless devices that enable dynamic home monitoring \\
& {$[34]$} \\
& - Improve understanding of urinary physiology by developing technologies \\
& to investigate concepts such as bladder micromotions [35] \\
& - Construct noninvasive methods to trigger micturition cascade through tran- \\
& scutaneous techniques [36,37] \\
& - Manufacture smaller devices on milli-, micro- and nano- scale [38] \\
& - Advance the development of systems to both sense bladder fullness and \\
Closed-Loop neuromodulation & stimulate voiding [39]
\end{tabular}

tients, as it involved destruction of intact nerves that controlled reflex erection and ejaculation [38]. This is an important issue for male patients. Therefore, other techniques, which avoided posterior rhizotomy and spared intact nerves were developed, but still usually involved open surgical implantation [39]. New treatment options attempt to overcome remaining obstacles in neuromodulation by using a transcutaneous approach instead of open procedures [40]. Through a transcutaneous approach, neuromodulation may be performed in a less technically complex, noninvasive, and cost-effective manner. Due to the current limited data, future studies, particularly those controlling for placebo effects, are indicated. Optimally, treatment for NLUTD after SCI involves the combination of sensor technology and neuromodulation for the development of a closed-loop system to monitor bladder parameters and stimulate micturition [41]. The development of these closed-loop systems will benefit greatly from identification of optimal biomarkers.

\section{Emerging discoveries}

Technical considerations to improve outcomes of bladder dysfunction and micturition continue to be explored in experimental animal models of SCI. These discoveries encompass portable and reliable urodynamic monitoring, percutaneous or implantable devices for specific neuromodulation of bladder control, and the combination of these elements into biofeedback devices to approach restored bladder function after SCI. As described, innovations in urodynamic testing aim to guide care management in real time for men with incomplete SCI. Several groups have recently used porcine models of bladder physiology to investigate submucosal implantation of sensors for localized bladder function [42]. Implantable sensors allow chronic monitoring of bladder parameters such as bladder wall acceleration and submucosal pressure as novel measures of detrusor muscle activity, as compared to urodynamic voiding cystography which is standard of care [42]. Alternative methods of noninvasive continuous monitoring complement urodynamic studies, but techniques such as infrared spectroscopy and ultrasound are affected by artificial testing conditions in a clinical environment and are limited to shorter duration of use [43].

Ambulatory urodynamic monitoring (AUM) is used to permit specific measurement of functional status in clinic. However, sensitivity and portability remain significant challenges since the aim of AUM is to reproduce bladder dysfunction in the clinic setting and requires connection to an external power source. For increased sensitivity, wearable telemetric devices would provide more relevant functional assessment during the course of a patient's daily activities [44]. Novel detection and treatment modalities are under development to enable real-time sensation of bladder filling for patients who are unable to perceive afferent sensory input in suprasacral SCI $[17,44]$. Nasrabadi and colleagues have recently described in detail the current status of wearable devices for urodynamic monitoring, which encompass methods such as ultrasound, near-infrared spectroscopy, body impedance, and implantable sensors [45]. Capsular intravesical devices are also under development to address concerns regarding treatment invasiveness, device migration, and erosion due to corrosive exposure to urine [46]. Preclinical studies to date have been trending towards wearable and non-invasive devices, but inherent challenges persist in portability and device alignment with varying body features [45]. The unique characteristics of each SCI insult has spurred progress towards the development of wearable and invasive technologies which may be tailored to patient-specific needs [45].

Real-time monitoring, notification, and subsequent intervention for increased intravesical pressure would enable timely voiding and minimize the incidence of complications in patients with SCI [17]. Treatment of SCI consequences including DSD has recently been studied in rat models of SCI by use of electrical nerve stimulation to restore bladder functionality, especially in those with complete injury [47]. Efferent stimulation to elicit detrusor muscle contraction also elicits the spinal guarding reflex and exter- 
nal urethral sphincter contraction, which impairs effective voiding $[17,47]$. A novel neuromodulation paradigm with low frequency electrical stimulation targeting distal pelvic nerves was performed simultaneously with proximal kilohertz high-frequency afferent nerve blockade. This combination was found effective for decoupling contraction of the detrusor muscle from reflex external urethral sphincter contraction, thereby improving voiding efficiency [47]. Though innovations in bladder management such as neuromodulation and continuous ambulatory urodynamic monitoring show great promise, current paradigms are designed for research purposes and remain limited in clinical use. Future study in large experimental models and clinical translation will require a detailed consideration of durability, invasiveness, treatment outcome, and most importantly patient experience to inform device development [46].

\section{Conclusions}

The management of NLUTD after SCI in males is continuing to evolve. Standard diagnostic tools are being optimized for widespread applicability. In this review, we highlighted the underlying anatomical contribution and current diagnostic strategies. We delved into current treatment approaches and highlighted the new innovations and emerging discoveries. Research is proceeding regarding improved treatment options in this patient population both for men with incomplete and complete SCI.

\section{Author contributions}

WD, KM, CS, KEVP, DP wrote individual sections. SM helped with figure. BLW lead author and idea generator. KENP helped critical editing.

\section{Ethics approval and consent to participate}

Not applicable.

\section{Acknowledgment}

Thanks to all the peer reviewers for their opinions and suggestions.

\section{Funding}

This research received no external funding.

\section{Conflict of interest}

The authors declare no conflict of interest.

\section{References}

[1] Hou S, Rabchevsky AG. Autonomic consequences of spinal cord injury. Comprehensive Physiology. 2014; 4: 1419-1453.

[2] DeVivo MJ. Epidemiology of traumatic spinal cord injury: trends and future implications. Spinal Cord. 2012; 50: 365-372.

[3] Milligan J, Goetz LL, Kennelly MJ. A Primary Care Provider's Guide to Management of Neurogenic Lower Urinary Tract Dysfunction and Urinary Tract Infection after Spinal Cord Injury. Topics in Spinal Cord Injury Rehabilitation. 2020; 26: 108-115.
[4] Vining RD, Gosselin DM, Thurmond J, Case K, Bruch FR. Interdisciplinary rehabilitation for a patient with incomplete cervical spinal cord injury and multimorbidity: a case report. Medicine. 2017; 96: e7837.

[5] Skeers P, Battistuzzo CR, Clark JM, Bernard S, Freeman BJC, Batchelor PE. Acute Thoracolumbar Spinal Cord Injury: Relationship of Cord Compression to Neurological Outcome. The Journal of Bone and Joint Surgery. American Volume. 2018; 100: $305-315$.

[6] Hamid R, Averbeck MA, Chiang H, Garcia A, Al Mousa RT, $\mathrm{Oh} \mathrm{S}$, et al. Epidemiology and pathophysiology of neurogenic bladder after spinal cord injury. World Journal of Urology. 2018; 36: $1517-1527$.

[7] Stoffel JT. Detrusor sphincter dyssynergia: a review of physiology, diagnosis, and treatment strategies. Translational Andrology and Urology. 2016; 5: 127-135.

[8] Adriaansen JJE, van Asbeck FWA, Tepper M, Faber WX, VisserMeily JMA, de Kort LMO, et al. Bladder-emptying methods, neurogenic lower urinary tract dysfunction and impact on quality of life in people with long-term spinal cord injury. The Journal of Spinal Cord Medicine. 2017; 40: 43-53.

[9] Dorsher PT, McIntosh PM. Neurogenic bladder. Advances in Urology. 2012; 2012: 816274.

[10] Bryk DJ, Zhao LC. Guideline of guidelines: a review of urological trauma guidelines. BJU International. 2016; 117: 226-234.

[11] Kessler TM, Traini LR, Welk B, Schneider MP, Thavaseelan J, Curt A. Early neurological care of patients with spinal cord injury. World Journal of Urology. 2018; 36: 1529-1536.

[12] Sekido N, Igawa Y, Kakizaki H, Kitta T, Sengoku A, Takahashi $\mathrm{S}$, et al. Clinical guidelines for the diagnosis and treatment of lower urinary tract dysfunction in patients with spinal cord injury. International Journal of Urology. 2020; 27: 276-288.

[13] Alsulihem A, Corcos J. Evaluation, treatment, and surveillance of neurogenic detrusor overactivity in spinal cord injury patients. Neuroimmunology and Neuroinflammation. 2019; 6: 13.

[14] Bright E, Cotterill N, Drake M, Abrams P. Developing a validated urinary diary: phase 1 . Neurourology and Urodynamics. 2012; 31: 625-633.

[15] Selius BA, Subedi R. Urinary retention in adults: diagnosis and initial management. American Family Physician. 2008; 77: 643-650.

[16] Liao L, Zhang F, Chen G. New grading system for upper urinary tract dilation using magnetic resonance urography in patients with neurogenic bladder. BMC Urology. 2014; 14: 38.

[17] Wyndaele J. The management of neurogenic lower urinary tract dysfunction after spinal cord injury. Nature Reviews. Urology. 2016; 13: 705-714.

[18] Wyndaele JJ. Intermittent catheterization: which is the optimal technique? Spinal Cord. 2002; 40: 432-437.

[19] Burns AS, Rivas DA, Ditunno JF. The Management of Neurogenic Bladder and Sexual Dysfunction after Spinal Cord Injury. Spine. 2001; 26: S129-S136.

[20] Lucas E. Medical Management of Neurogenic Bladder for Children and Adults: a Review. Topics in Spinal Cord Injury Rehabilitation. 2019; 25: 195-204.

[21] Kakizaki H, Ameda K, Kobayashi S, Tanaka H, Shibata T, Koyanagi $T$. Urodynamic effects of alpha1-blocker tamsulosin on voiding dysfunction in patients with neurogenic bladder. International Journal of Urology. 2003; 10: 576-581.

[22] Chancellor MB, Erhard MJ, Hirsch IH, Stass WE. Prospective evaluation of terazosin for the treatment of autonomic dysreflexia. The Journal of Urology. 1994; 151: 111-113.

[23] Madhuvrata P, Singh M, Hasafa Z, Abdel-Fattah M. Anticholinergic drugs for adult neurogenic detrusor overactivity: a systematic review and meta-analysis. European Urology. 2012; 62: 816-830. 
[24] Sager C, Sanmartino M, Burek C, Gomez YR, Vazquez Patiño $\mathrm{M}$, Weller S, et al. Efficacy and safety of Mirabegron as adjuvant treatment in children with refractory neurogenic bladder dysfunction. Journal of Pediatric Urology. 2020; 16: 655.e1655.e6.

[25] El Helou E, Labaki C, Chebel R, El Helou J, Abi Tayeh G, Jalkh $\mathrm{G}$, et al. The use of mirabegron in neurogenic bladder: a systematic review. World Journal of Urology. 2020; 38: 2435-2442.

[26] Thaker H, Zhang S, Diamond DA, Dong M. Beyond botulinum neurotoxin a for chemodenervation of the bladder. Current Opinion in Urology. 2021; 31: 140-146.

[27] Martens FMJ, Sievert K. Neurostimulation in neurogenic patients. Current Opinion in Urology. 2020; 30: 507-512.

[28] Sauerwein D. Surgical treatment of spastic bladder paralysis in paraplegic patients. Sacral deafferentation with implantation of a sacral anterior root stimulator. Der Urologe. Ausg. A. 1990; 29: 196-203.

[29] Van Kerrebroeck PE, Koldewijn EL, Rosier PF, Wijkstra H, Debruyne FM. Results of the treatment of neurogenic bladder dysfunction in spinal cord injury by sacral posterior root rhizotomy and anterior sacral root stimulation. The Journal of Urology. 1996; 155: 1378-1381.

[30] Martens FMJ, Heesakkers JPFA. Clinical results of a brindley procedure: sacral anterior root stimulation in combination with a rhizotomy of the dorsal roots. Advances in Urology. 2011; 2011: 709708

[31] Hohenfellner M, Pannek J, Bötel U, Dahms S, Pfitzenmaier J, Fichtner J, et al. Sacral bladder denervation for treatment of detrusor hyperreflexia and autonomic dysreflexia. Urology. 2001; 58: $28-32$.

[32] Samson G, Cardenas DD. Neurogenic Bladder in Spinal Cord Injury. Physical Medicine and Rehabilitation Clinics of North America. 2007; 18: 255-274.

[33] Wille S, Schumacher P, Paas J, Tenholte D, Eminaga O, Müller $\mathrm{U}$, et al. Catheterless long-term ambulatory urodynamic measurement using a novel three-device system. PLoS ONE. 2014; 9: e96280.

[34] Liu L, Zhao K, Chen F, Wu J, Yang Z, Chen M, et al. Testing of a New Portable Device for Dynamic Bladder Pressure Monitoring. Lower Urinary Tract Symptoms. 2018; 10: 193-197.

[35] Soebadi MA, Weydts T, Brancato L, Hakim L, Puers R, De Ridder D. Novel implantable pressure and acceleration sensor for bladder monitoring. International Journal of Urology. 2020; 27 : 543-550.

[36] Parittotokkaporn S, Varghese C, O’Grady G, Lawrence A, Svirskis D, O'Carroll SJ. Transcutaneous Electrical Stimulation for Neurogenic Bladder Dysfunction Following Spinal Cord Injury: Meta-Analysis of Randomized Controlled Trials. Neuromodulation. 2021; 24: 1237-1246.

[37] de Abreu GE, de Souza LA, da Fonseca MLV, Barbosa TBC, de Mello ERD, Nunes ANB, et al. Parasacral Transcutaneous Electrical Nerve Stimulation for the Treatment of Children and Adolescents with Bladder and Bowel Dysfunction: a Randomized Clinical Trial. Journal of Urology. 2021; 205: 1785-1791.

[38] Darrow D, Balser D, Netoff TI, Krassioukov A, Phillips A, Parr A, et al. Epidural Spinal Cord Stimulation Facilitates Immediate Restoration of Dormant Motor and Autonomic Supraspinal Pathways after Chronic Neurologically Complete Spinal Cord Injury. Journal of Neurotrauma. 2019; 36: 2325-2336.

[39] Park E, Lee J, Kang M, Cho K, Cho BH, Lee K. Detecting Bladder Biomarkers for Closed-Loop Neuromodulation: a Technological Review. International Neurourology Journal. 2018; 22: 228-236.

[40] Schäfer W, Abrams P, Liao L, Mattiasson A, Pesce F, Spangberg A, et al. Good urodynamic practices: uroflowmetry, filling cystometry, and pressure-flow studies. Neurourology and Urodynamics. 2002; 21: 261-274.

[41] Yu L, Kim BJ, Meng E. Chronically implanted pressure sensors: challenges and state of the field. Sensors. 2014; 14: 20620 20644.

[42] Kirkham APS, Knight SL, Craggs MD, Casey ATM, Shah PJR. Neuromodulation through sacral nerve roots 2 to 4 with a Finetech-Brindley sacral posterior and anterior root stimulator. Spinal Cord. 2002; 40: 272-281.

[43] Yiou R, Audureau E, Loche C, Dussaud M, Lingombet O, Binhas M. Comprehensive evaluation of embarrassment and pain associated with invasive urodynamics. Neurourology and Urodynamics. 2015; 34: 156-160.

[44] Abelson B, Majerus S, Sun D, Gill BC, Versi E, Damaser MS. Ambulatory urodynamic monitoring: state of the art and future directions. Nature Reviews Urology. 2019; 16: 291-301.

[45] Nasrabadi MZ, Tabibi H, Salmani M, Torkashvand M, Zarepour E. A comprehensive survey on non-invasive wearable bladder volume monitoring systems. Medical and Biological Engineering and Computing. 2021; 59: 1373-1402.

[46] Basu AS, Majerus S, Ferry E, Makovey I, Zhu H, Damaser MS. Is submucosal bladder pressure monitoring feasible? Proceedings of the Institution of Mechanical Engineers. 2019; 233: 100 113.

[47] Peh WYX, Mogan R, Thow XY, Chua SM, Rusly A, Thakor $\mathrm{NV}$, et al. Novel Neurostimulation of Autonomic Pelvic Nerves Overcomes Bladder-Sphincter Dyssynergia. Frontiers in Neuroscience. 2018; 12: 186. 